\title{
EXPERIENCIA DE APRENDIZAJE EN LA TRANSMISIÓN DE \\ CONOCIMIENTOS TEÓRICOS Y PRÁCTICOS CON APLICACIÓN DE UNA \\ EVALUACIÓN FORMATIVA EN LABORATORIOS DE QUÍMICA
}

Learning experience of theoretical and practical knowledge with application of

formative assessment in chemistry laboratories

Experiência de aprendizagem de conhecimentos teóricos e práticos com aplicação de avaliação formativa em laboratórios de química

Víctor Álvarez Valverde (1)

Priscilla Hernández Elizondo (2)

(1) Universidad Nacional, Costa Rica. Teléfono +506 22773271. Correo electrónico: victor.alvarez.valverde@una.cr

(2) Universidad de Costa Rica, Costa Rica. Teléfono +506 88992229. Correo

electrónico:pris23@gmail.com

\begin{abstract}
Resumen
En los laboratorios de Química General, Química Orgánica y Bioquímica, se ha dado un estricto énfasis a la verificación del conocimiento adquirido por los estudiantes, a través de métodos tales como la preparación previa, -tanto de alumnos, como de docentes-; realización de pruebas cortas, discusión de la materia antes de iniciar el laboratorio y, en forma grupal, al finalizar la práctica. Asimismo, los estudiantes deben elaborar un informe en el que expliquen, con apoyo del material bibliográfico actualizado, su interpretación de lo ocurrido al culminar la experiencia. Estas acciones despiertan su interés a lo largo del semestre, lo cual se traduce en una elevada promoción.

Palabras clave: prácticas en laboratorios; metodologías de evaluación; evaluación formativa
\end{abstract}

\begin{abstract}
Laboratories of General Chemistry, Organic Chemistry and Biochemistry are subjects in which, reiterative verification of concepts and laboratory skills as a formative assessment have played an important role in the learning process. This has been Experiencia de aprendizaje en la transmisión de conocimientos teóricos y prácticos con aplicación de una evaluación formativa en laboratorios de química


accomplished by: earlier preparation of both, teachers and students for the laboratory lessons; the realization of short exams before the laboratory session; a discussion on the practice at the beginning and at the end of the laboratory session and the completion of a laboratory inform in which the students, supported by updated bibliography references, explain, on their own words, the results obtained during the laboratory session. Additionally, teachers regularly discuss their experiences during the laboratory session in order to improve the quality of the practice. Those previous actions have stimulated the interest of the students; showing an important student promotion at the end of the semester.

Keywords: practices in laboratories; evaluation methodologies; formative assessment

\section{Resumo}

As aulas práticas dos cursos de química geral, química orgânica e bioquímica são práticas que utilizam de forma repetitiva os métodos de verificação de conhecimentos adquiridos pelos alunos. O anterior é resultado de uma preparação prévia tanto dos estudantes como dos professores, além da realização de provas curtas e espaços de discussão sobre o assunto antes de iniciar as aulas práticas. Também como conseqüência da formação de grupos de discussão ao final das aulas e elaboração de relatórios por parte dos estudantes, nos quais são relatadas, apoiados por material bibliográfico recente, sua interpretação do acontecido durante a prática. Essas ações têm atraído o interesse dos estudantes, os quais se vêm motivados durante o semestre, gerando ao final uma excelente taxa de aprovação do curso em semestres consecutivos.

Palavras-chave: aulas práticas, metodologias de avaliação, avaliação formativa

\section{Introducción}

La orientación académica de cualquier disciplina debe proyectarse de manera integral y, en el caso de la enseñanza superior de las asignaturas de Química, esta no es la excepción. El abordaje de su estudio debe contemplar aspectos teóricos detallados y destrezas prácticas.

Los contenidos teóricos de la Química se desarrollan a través de las asignaturas de corte magistral, mientras que la modalidad de laboratorios otorga a los estudiantes Experiencia de aprendizaje en la transmisión de conocimientos teóricos y prácticos con aplicación de una evaluación formativa en laboratorios de química 
las destrezas prácticas para su comprobación. Por su parte, la evaluación formativa y sus muchas posibilidades de aplicación, brindan al alumno una experiencia de aprendizaje más integral; sin embargo, estas estrategias deben ser ajustadas a cada asignatura particular para poder obtener óptimos resultados de aprendizaje.(Fraile, López-Pastor, Castejón \& Romero, 2013).

Los procesos de comprobación de destrezas o conocimientos teóricos específicos por parte del docente, son en ocasiones complejos, por lo que es menester que se lleven a cabo de manera constante, dinámica y tomando en consideración las particularidades de aprendizaje de cada persona en el proceso. Esta labor ha permitido que se impacte positivamente al estudiantado ya que se estimula la retroalimentación permanente entre docentes y alumnos (Vogelzan \& Admiraal, 2017).

\section{Contextualización}

En este caso, las asignaturas seleccionadas son: Laboratorio de Química General (4 grupos de 18 estudiantes); Química Orgánica (4 grupos de 18 estudiantes) y Bioquímica (3 grupos de 18 estudiantes), todas ellas forman parte de titulaciones a nivel de grado universitario, tales como Ingeniería Agronómica, Biología, Enseñanza de las Ciencias y Química. Estas materias se imparten bajo la modalidad de laboratorios semestrales e involucran estudiantes de distintas etapas de su formación universitaria (inicial, intermedia y final respectivamente). El trabajo realizado lo desempeñan mediante una evaluación de carácter individual, complementado con uno de carácter cooperativo, donde se ejecutan técnicas para el desarrollo de destrezas prácticas que complementen lo visto en las lecciones teóricas.

\section{Diseño y desarrollo}

$\mathrm{Al}$ iniciar el semestre los estudiantes son debidamente informados a través de la entrega de la carta o programa, acerca de los detalles de la constitución, evaluación y calendarización de la asignatura, en ellos se incluye la documentación y guía didáctica de los laboratorios que se desarrollarán durante ese periodo, los cuales normalmente varían entre los 12 y 14, según la materia.

En general, las estaciones de trabajo están dispuestas en parejas. Al iniciar cada sesión, el docente enfatiza los objetivos y contenidos relacionados con la práctica de ese día y durante las restantes 2 horas de clase, los estudiantes desarrollan los Experiencia de aprendizaje en la transmisión de conocimientos teóricos y prácticos con aplicación de una evaluación formativa en laboratorios de química 
procedimientos y completan las libretas de laboratorio. Al final del laboratorio, se discute de forma compartida, participativa y democrática los resultados obtenidos y la retroalimentación del día.

A través de una prueba corta, los estudiantes son evaluados acerca de los contenidos necesarios para la clase; seguidamente hay un conversatorio grupal sobre el fundamento teórico que se utilizará en el desarrollo de cada experimento. Cada pareja es supervisada sistemáticamente, con tablas de cotejo, mientras realizan su práctica. La interacción profesor-alumno es constante con la finalidad de regular periódicamente el ritmo de aprendizaje.

\section{Evaluación}

La evaluación formativa de los aprendizajes en un laboratorio de Química, implica estar constantemente valorando conceptos y destrezas. Mediante una prueba corta, se corrobora que los estudiantes se hayan preparado conceptual y procedimentalmente para la ejecución de la práctica o el experimento de laboratorio.

Durante la evaluación formativa de las destrezas, los alumnos son puestos a prueba mediante la manipulación de muestras, equipo de laboratorio y cristalería de diversa índole. Esto les permite interactuar de manera personalizada con los procesos básicos que se ejecutan en un laboratorio de Química.

Cada pareja es supervisada sistemáticamente con tablas de cotejo mientras realizan su práctica. La interacción profesor-alumno es constante, y para complementar la evaluación formativa, durante los últimos veinte minutos de la clase, se discuten de forma compartida, participativa y democrática los resultados obtenidos y la retroalimentación del día.

La discusión final pretende que dicha experiencia formativa, en adelante, se integre al conjunto de conocimientos generados a lo largo del semestre y complemente contenidos adquiridos en cursos anteriores, los cuales han sido definidos como requisitos para poder participar en cada uno de los laboratorios en cuestión.

Una vez concluida cada sesión de laboratorio, los estudiantes deben presentar un informe, con apoyo de material bibliográfico actualizado, en el que se refleje lo experimentado y aprendido durante dicha sesión y se permita discutir e interpretar los 
resultados obtenidos; esto permite retomar los conceptos en más de una ocasión tanto dentro como fuera del laboratorio.

Otro aspecto destacable, es el continuo debate que surge entre los docentes que imparten los laboratorios, lo cual genera una retroalimentación que permite controlar mejor las variables que acontecen en cada sesión de trabajo (Henderson, Turpen, Dancy \& Chapman, 2014).

\section{Conclusiones}

1. La reiteración de conceptos y destrezas en un laboratorio de Química, mediante la ejecución de diversas herramientas, permite evaluar si la instrucción fue efectiva en el estudiante.

2. La implementación de un seguimiento relativamente personalizado a todos los alumnos a lo largo de un semestre, implica por parte de los docentes, una carga de trabajo mayor a la convencional.

3. Es importante resaltar que, en general, ha existido una buena disposición por parte del estudiantado a la utilización de las estrategias descritas anteriormente, lo cual se refleja en las altas tasas de aprobación que presentan estas asignaturas de laboratorio.

\section{Referencias}

Fraile, A., López-Pastor, V., Castejón, J., \& Romero, R. (2013). La evaluación formativa en docencia universitaria y el rendimiento académico del alumnado. Aula Abierta, 41(2), 23-34.

Henderson, C., Turpen, C., Dancy, M., \& Chapman, T. (2014). Assessment of teaching effectiveness: Lack of alignment between instructors, institutions, and research recommendations. Physical Review Special Topics - Physics Education Research, 10(1), 1-20.

Vogelzan, J., \& Admiraal, W. (2017). Classroom action research on formative assessment in a context-based chemistry course. Journal of Educarional Action Research, 25(1), 155-166.

Experiencia de aprendizaje en la transmisión de conocimientos teóricos y prácticos con aplicación de una evaluación formativa en laboratorios de química 